\title{
Beyond Weakly-supervised: Pseudo Ground Truths Mining for Missing Bounding-boxes Object Detection
}

\author{
Yongqiang Zhang, Mingli Ding*, Yancheng Bai, Mengmeng Xu, and Bernard Ghanem
}

\begin{abstract}
Due to the shortcomings of the weakly-supervised and fully-supervised object detection (i.e. unsatisfactory performance and expensive annotations, respectively), leveraging partially labeled images in a cost-effective way to train an object detector has attracted much attention. In this paper, we formulate this challenging task as a missing bounding-boxes object detection problem. Specifically, we develop a pseudo ground truth mining (PGTM) procedure to automatically find the missing boundingboxes for the unlabeled instances, called pseudo ground truths here, in the training data, and then combine the mined pseudo ground truths and the labeled annotations to train a fullysupervised object detector. Furthermore, we further propose an incremental learning (IL) framework to gradually incorporate the results of the trained fully-supervised detector to improve the performance of missing bounding-boxes object detection. More importantly, we find an effective way to label the massive images with limited labors and funds, which is crucial when building a large-scale weakly/webly labeled dataset for object detection. Extensive experiments on the PASCAL VOC and COCO benchmarks demonstrate that our proposed method can narrow the gap between fully-supervised and weakly-supervised object detectors, and we outperform the previous state-of-the-art weakly-supervised detectors by a large margin (more than $3 \%$ mAP absolutely) when the missing rate equals 0.9. Moreover, our proposed method with $30 \%$ missing bounding-box annotations can achieve comparable performance to some fully-supervised detectors.
\end{abstract}

Index Terms-Pseudo ground truths, weakly/semi-supervised, object detection, missing bounding-boxes.

\section{INTRODUCTION}

$\mathbf{O}$ BJECT detection is an important and fundamental task in computer vision, which aims to localize the position of each instance and classify the category of each target object. Moreover, it is a vital step towards many real-world applications [1]-[3], like image retrieval, intelligent surveillance, autonomous driving, etc. . Object detection has been widely studied in the past few decades, and significant progress has been made with the success of deep convolutional neural networks (DCNNs) [4]-[6].

Currently, the state-of-the-art object detection methods [7][17] are based on the fully-supervised learning (FSL) techniques, which usually train detection networks [4], [18] on the

Yongqiang Zhang and Mingli Ding are with School of Electrical Engineering and Automation, Harbin Institute of Technology (HIT), Harbin, China (Email: yongqiang.zhang.hit@gmail.com, Mingli.ding.hit@gmail.com).

Mengmeng $\mathrm{Xu}$, Yancheng Bai and Bernard Ghanem are with Visual Computing Center, King Abdullah University of Science and Technology (KAUST), Thuwal, Saudi Arabia (Email: mengmengxu@kaust.edu.sa, Yancheng.Bai.1987@gmail.com, Bernardghanem@gmail.com).

* Corresponding author. large scale benchmarks (e.g. PASCAL VOC [19], COCO [20]) with instance-level annotations (i.e. bounding-boxes). The key factor to their finer performance is the strong learning ability of the fully-supervised DCNNs and the availability of large scale labeled datasets with tight bounding-box annotations. However, the main shortcoming of these methods is that they require the accurate instance-level annotation for each object, which is extremely expensive and time-consuming.

To address the aforementioned issue, some works [1], [2], [21]-[24] try to utilize the weakly-supervised learning (WSL) techniques to train a weakly-supervised detector (WSD) only under the supervision of image-level. The motivation behind these methods is that it is much easier to build a large scale benchmark with only image-level labels than to compile one with the accurate bounding-box annotations. However, we would like to note that the performance of WSDs are far behind the fully-supervised detectors (FSDs). This shortcoming (i.e. unsatisfactory performance) hinders the application of WSDs in some practical scenarios.

As to the disadvantages of the fully-supervised and weaklysupervised object detection methods, we would like to ask: is there a middle ground that can reduce the cost of instancelevel annotations while achieving an accurate object detector with satisfactory performance. A natural solution to this question is using images with partially labeled instance-level annotations to train an object detector. Some existing methods [25]-[34] treat it as a semi-supervised problem, and employ the semi-supervised learning (SSL) techniques to solve it. However, these SSL based methods usually make use of the visual or semantic knowledge transfer technique to find the pseudo ground truth bounding-boxes for the unlabeled instances, which is complicated and impractical for large-scale object detection, especially for those hard images (i.e. images including multiple objects and some of them are occluded).

Attempting to overcome the above-motioned drawbacks of the existing semi-supervised object detectors, in this paper, we formulate object detection supervised under the partially labeled data as a missing bounding-boxes object detection problem. To be specific, we develop a pseudo ground truth mining (PGTM) procedure to automatically find the missing bounding-boxes for the unlabeled instances. Our proposed PGTM procedure is motivated by the recent success of the weakly to fully supervised learning techniques [1], [2], [24]. Instead of only relying on the image-level labels to train an object detector, we try to combine a small number of instance-level annotations labeled by humans for improving 

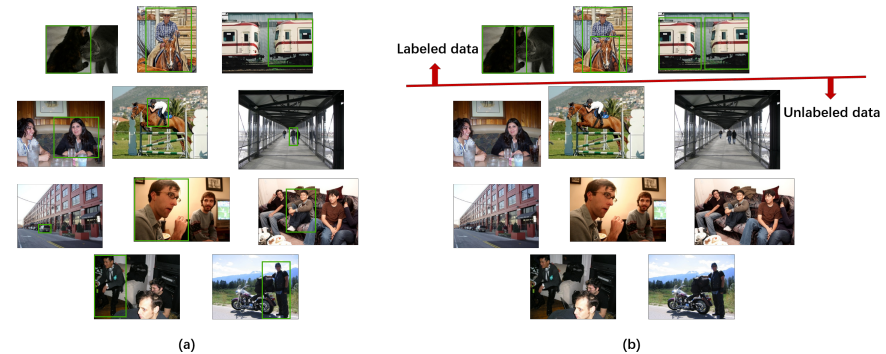

Fig. 1. Different settings of the partially labeled instance-level annotations. In (a), each image in the training data has instance-level annotations, but only the obvious (or simple) instances are labeled. In (b), only part of images in the training data have instance-level annotations where all instances in these images are annotated, and other images have no instance-level annotations. Best seen on the computer and zoomed in.

the detection performance. Our basic idea is that given the training data with partially labeled instance-level annotations, we first adopt the proposed PGTM algorithms to predict the bounding-boxes for all instances, which are treated as the pseudo ground truths in the following steps, and then these mined pseudo ground truth bounding-boxes and the partially labeled instance-level annotations are combined to train an object detector. For this purpose, we propose two combination methods (i.e. hard/soft combination method), and fine details can be found in subsection IV-B. To reinforce the quality of the mined pseudo ground truth bounding-boxes, we further propose an incremental learning (IL) framework to gradually incorporate the results of the trained FSD.

Another motivation for viewing object detection supervised under the partially labeled data as a missing bounding-boxes object detection problem is that we want to investigate the impact of missing instance-level annotations on an object detector and further reveal that how to build a large-scale benchmark with limited labors and funds. There are two different settings for this purpose: (1) each image in the training data has instance-level annotations, but only the obvious/simple instances are labeled, as shown in Figure 1 (a); (2) only part of images in the training data have instance-level annotations (i.e. all instances in these images have instancelevel annotations), and other images have no instance-level annotations, as shown in Figure 1 (b).

To sum up, we make the following four main contributions in this work: (1) we formulate the task of object detection supervised under the partially labeled data as a missing bounding-boxes object detection problem, and we develop a novel algorithm, named pseudo ground truth mining (PGTM) procedure, to automatically find the missing bounding-boxes for the unlabeled instances in the training data. (2) we further propose a new incremental learning (IL) framework to gradually incorporate the results of the trained detector to improve the performance of the missing bounding-boxes object detection. (3) we reveal an effective way to label the massive images with limited labors and funds, which is crucial when building a large scale weakly/webly dataset for object detection. (4) Finally, extensive experiments on the PASCAL VOC and COCO benchmarks demonstrate that our method can narrow the gap between fully-supervised and weakly- supervised object detectors, and we outperform the previous state-of-the-art weakly-supervised detectors by a large margin. Furthermore, our method with $30 \%$ missing bounding-boxes can achieve comparable performance to some fully-supervised detectors.

\section{RELATED WORK}

\section{A. Fully-supervised Object Detection}

In this paper, we focus on reviewing the related works about CNNs-based methods, which can be divided into two categories: one-stage framework and two-stage framework.

In the one-stage framework, the detector directly classifies the image regions or anchors into specific categories and regresses bounding-boxes in a dense manner. For instance, YOLO [16] divides the input image into an $S \times S$ grid and uses the whole topmost feature map to directly predict confidences of multiple categories and bounding-boxes. SSD [14] discretizes the output space of bounding-boxes into a set of anchors, and the trained network generates the score for the presence of each object category and produces adjustment for each anchor to better match the object location. To further speed up the one-stage detectors and improve the detection performance, some YOLO follow-up works [17], [35] and some SSD subsequent methods [15], [36], [37] have been proposed over the next few years.

In the two-stage framework, there are two separate steps: the first one is proposal generation and the second one is post-classification and regression. Region-based CNN (i.e. RCNN [9]) is a milestone of the two-stage object detector. In R-CNN [9], the region proposals are generated by Selective Search [38], and each region proposal is processed separately by the DCNN to learn the features for classification and regression, which is very time-consuming. Towards this problem, ROI-Pooling is proposed in SPPnet [39] and introduced in Fast-RCNN [8], thus improving the efficiency greatly. But the proposal generation is a separate operation in Fast-RCNN, which is annoying. By training both of these two stages in an end-to-end way, Faster-RCNN [12] achieves a satisfactory result in both detection performance and computational efficiency. Over time, some RCNN-based follow-up works [10], [13], [40], [41] are proposed to further improve the detection performance and to speed up the computational efficiency.

Compared to the fully-supervised object detection methods, we do not need the accurate instance-level annotation for each instance, and only using few labeled instance-level annotations can achieve comparable performance to some FSDs.

\section{B. Weakly-supervised Object Detection}

Weakly-supervised methods try to learn classification and localization information from image-level labels, and do not need bounding-box annotations. Recently, some CNNsbased approaches [1], [23], [24], [26], [42]-[44] for weaklysupervised object detection have been proposed. [23] proposes a weakly-supervised deep detection network (WSDDN), which is a two-stream architecture and the positive samples are selected by multiplying the score of the output of two streams. [42] attempts to localize the instance by using class activation 
maps (CAM) generated from a classification trained CNN. [24] improves the performance by reforming the optimization strategy, and they design an online instance classifier refinement algorithm to alleviate the local optimum. [1] proposes the pseudo ground-truth excavation (PGE) and pseudo groundtruth adaptation (PGA) algorithms to mine the pseudo ground truth bounding-box for each instance, and then uses the mined pseudo ground truths to train an FSD. [43] and [26] propose an adversarial erasing approach for localizing and expanding object regions progressively.

Actually, the performance of WSDs is far from satisfaction, and there is a large gap when comparing with the performance of FSDs. In this paper, we improve the performance of WSDs by incorporating a few labeled instances to train a missing bounding-boxes object detection network.

\section{Semi-supervised Object Detection}

In the setting of the semi-supervised learning, a small amount of fully labeled data and a large amount of weakly labeled (or unlabeled) data are provided. Some methods [45][47] for object detection have been proposed, and most of these methods treat it under the semi-supervised setting as a transfer learning [48] problem. Specifically, they assume that some strongly annotated categories exist, and try to transfer the visual or semantic knowledge from these strongly annotated categories to the weakly annotated categories. [49] proposes an appearance transfer approach by transferring semantic knowledge from the familiar objects to localize the boundingboxes of the novel objects. [50] transfers the tracked location of objects from the labeled videos to generated the boundingboxes for the weakly-labeled images. [47] improves the previous works by adopting the knowledge of object similarities from semantic and visual domains during the transfer process.

Different from these methods, we focus on using the imagelevel labels and the partially instance-level annotations, which are in the same dataset, to solve the problem of missing bounding-boxes object detection, and no additional strongly annotated data are required in our paper.

\section{WHAT IS THE IMPACT OF MISSING BOUNDING-BOXES FOR OBJECT DETECTION?}

\section{A. Preliminaries}

Dataset and evaluation metrics. Pascal VOC 2007 and VOC 2012 are two widely used benchmarks in the object detection task, in which 9,963 and 22,531 images from 20 object categories are included, respectively. Both image-level labels and instance-level annotations are provided in the datasets. For VOC 2007, we use the trainval set for training and use the test set for testing. For VOC 2012, we choose the trainval set to train our detectors and evaluate on the test set. We follow the standard metrics for object detection on Pascal VOC to report the detection results, i.e. mean average precision (mAP) [19] is used as the evaluation metric to evaluate our trained model, where a correct detection result has an $\mathrm{IoU}>$ 0.5 with the annotated ground truth bounding-boxes.

Detectors. To prove that missing bounding-boxes have a serious impact on all existing object detectors, we choose four popular detectors in all our experiments. These detectors can be divided into two categories: two-stage framework and one-stage framework. In the two-stage framework, we choose the commonly used Fast-RCNN [8] and Faster-RCNN [12] as our baseline, and then train them by using the missing bounding-box annotations at different missing rates. In the one-stage framework, we adopt the two most popular detectors (i.e. SSD [14] and YOLO [16]) to verify the influence of the missing bounding-boxes on the results of object detection.

\section{B. Missing Bounding-boxes Generation}

Based on the ground truth bounding-box annotations labeled by humans on Pascal VOC datasets, we generate the missing bounding-box annotations by randomly dropping some bounding-boxes at different missing rates. In this paper, we provide two different methods for generating the missing bounding-box annotations: (i) image-level missing annotations, and (ii) instance-level missing annotations.

Image-level Missing Annotations. The simplest way to generate the missing bounding-box annotations is to discard the annotations of some images directly, and save the annotations of others, as shown in Figure 1 (b).

Instance-level Missing Annotations. Another way to generate the missing bounding-box annotations is to discard the bounding-boxes of each category at different missing rates. Specifically, we collect all instance-level annotations of the training data for each category, and then randomly discard some bounding-boxes at the different missing rates, as shown in Figure 1 (a). Finally, we save the remaining bounding-boxes to train the above mentioned FSDs.

\section{Analysis}

Missed detection. We investigate the results of the detectors trained under the supervision of missing bounding-box annotations, and find that the majority of failure cases are false negatives (i.e. some instances are missed and detected as backgrounds). The missed detection problem is particularly serious in the crowded scenarios.

In Figure 2, we report the detection performance of four commonly used detectors (i.e. Faster-RCNN [12], FastRCNN [8], SSD [14], YOLO [16]), which are trained by using the missing bounding-box annotations generated by the instance-level missing method on Pascal VOC 2007 and VOC 2012 benchmarks, respectively. We observe that the detection performance (mAP) of all detectors drops significantly from $\sim 70 \%$ to $\sim 30 \%$ when the missing rate increases, indicating that the missing bounding-boxes seriously degrade the performance of object detectors. Surprisingly, the performance of all detectors trained with the missing bounding-box annotations at a high missing rate is worse than an WSD [1], as the dot line shown in Figure 2 (a) and Figure 2 (b).

Conclusion. The above results validate the missing bounding-boxes have a serious impact on object detectors, and the detection performance is even worse than an WSD as the missing rate increases. To address this issue, in Section IV, an PGTM procedure is proposed to mine the pseudo ground truth bounding-boxes for the unlabeled instances (i.e. missing 


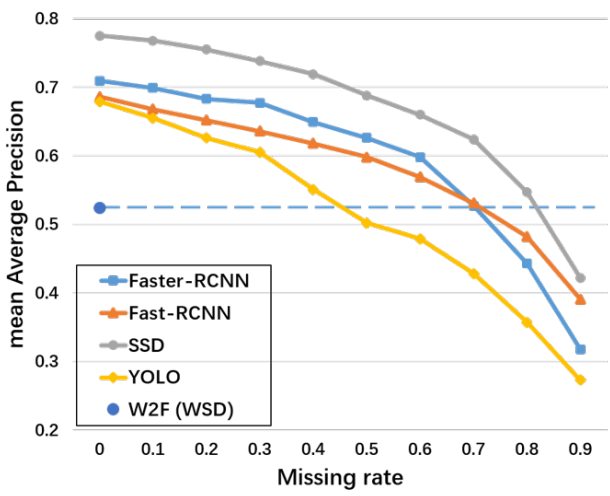

(a) Pascal VOC 2007

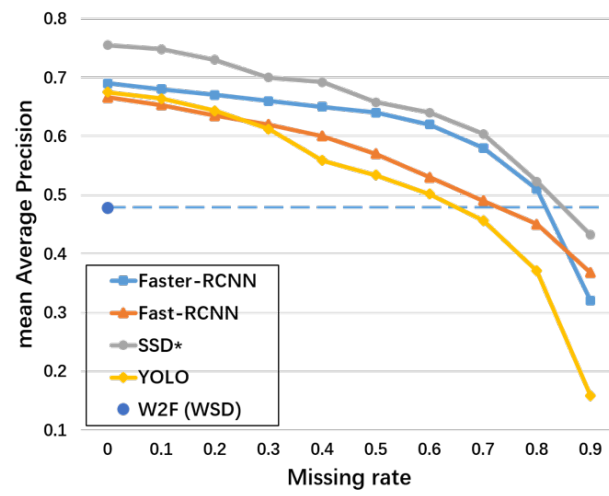

(b) Pascal VOC 2012

Fig. 2. The detection performance (mAP) on some popular fully-supervised detectors under different missing bounding-boxes settings, and on a weaklysupervised detector (i.e. W2F) trained by only image-level labels. In (a), all the detectors are trained on Pascal VOC 2007 trainval set, and tested on Pascal VOC 2007 test set. In (b), all the detectors are trained on Pascal VOC 2012 trainval sets, and tested on Pascal VOC 2012 test set. Following [14], SSD* is trained on Pascal VOC 2007 trainval, test, and Pascal VOC 2012 trainval sets, and tested on Pascal VOC 2007 test set.

bounding-boxes) in the training data, and an IL framework is proposed to gradually incorporate the results of the trained FSD for improving the performance of missing boundingboxes object detection.

\section{Proposed Method}

\section{A. Revisiting $W 2 F$}

Weakly-supervised object detection offers a reasonable way to detect instances by only using the image-level labels. [1], [24] propose a weakly to fully supervised framework which can detect the objects by first training an WSD under the supervision of only image-level labels (i.e. no instance-level annotations are available), and then training an FSD by using the pseudo ground truth bounding-boxes generated from the trained WSD. In this paper, we follow their method in [24] to train our WSD.

After training the WSD, the candidate bounding-box regions are generated from the trained WSD. Meanwhile, a postprocessing method is proposed to mine the pseudo ground truth bounding-boxes in W2F [1], which are further used to train an FSD. However, the achieved pseudo ground truth bounding-boxes are still low-quality since no ground truth bounding-box annotations are available in their method. In sub-section IV-B, based on the few instance-level annotations, we develop a pseudo ground truth mining (PGTM) procedure to automatically find the high-quality pseudo ground truth bounding-boxes for the unlabeled instances.

\section{B. The Proposed PGTM Algorithm}

In this paper, we assume that the bounding-boxes of the unlabeled instances are missing, and we propose an PGTM procedure to generate the high-quality pseudo ground truth bounding-boxes for those unlabeled instances. The basic idea for the missing bounding-boxes object detection is that we train an WSD as described in subsection IV-A to generate the candidate bounding-box regions, and then combine the pseudo ground truth bounding-boxes generated by the PGTM and the partially labeled bounding-boxes to train an FSD.
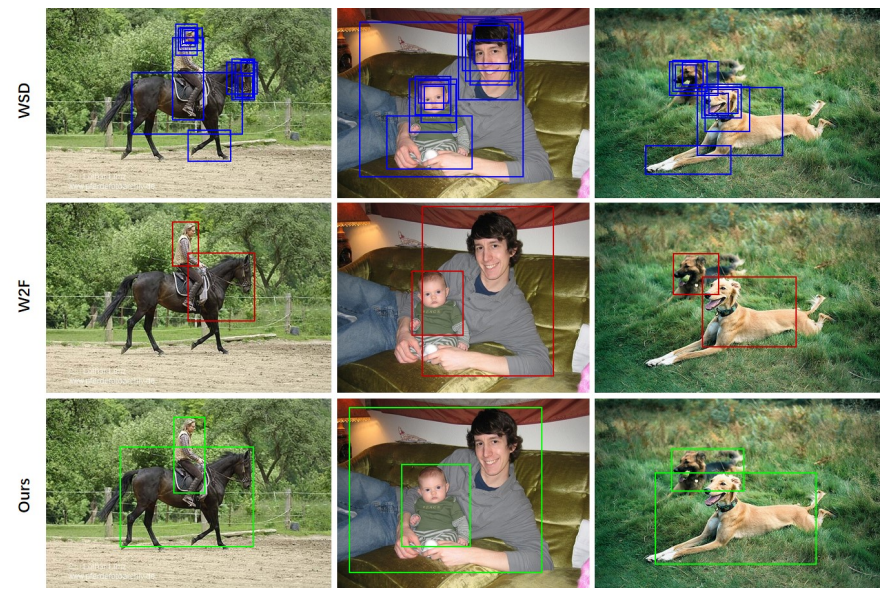

Fig. 3. The predictions of the WSD, and the comparison of methods (i.e. $\mathrm{W} 2 \mathrm{~F}$ [1] and ours) for generating the pseudo ground truth boundingboxes. Obviously, the quality of the pseudo ground truth bounding-boxes generated by our methods are better than W2F [1]. Best seen on the computer, in color and zoomed in.

Different from [1], which excavates and adapts the pseudo ground truth bounding-boxes by only using the predictions from the WSD, we utilize the partially labeled boundingboxing and the predictions from the WSD to further refine mined bounding-boxes by the proposed PGTM procedure.

The bounding-boxes predicted by the trained WSD only highlight small regions of target objects instead of entire objects, as shown in Figure 3, which are far from the requirement of the accurate ground truth bounding-boxes. Our proposed PGTM aims at discovering the tightly boundingboxes for target objects. In particular, three procedures are used to mine the accurate pseudo ground truth boundingboxes: (i) we apply the non-maximum suppression (NMS) operation with a threshold $T_{n m s}=0.3$ on all the predictions from the WSD, and only save the bounding-boxes with scores larger than a pre-defined threshold (i.e. $T_{\text {score }}$ ) 0.2 as the candidate pseudo ground-truth bounding-box regions. (ii) The discriminative bounding-boxes predicted by the WSD usually 
have a high score, and are usually completely surrounded by the tight bounding-boxes with a low score. We want to remove these discriminative bounding-boxes in this step. To be specific, we first choose the biggest bounding-box from the predictions from the WSD, delete all the discriminative bounding-boxes contained in this chose bounding-box, and save this biggest bounding-box. After that, we select the second biggest bounding-box and do the same process, and so on. Step ii prevents the small discriminative regions being chosen as a pseudo ground truth bounding-box. (iii) Some instances may not have a very tight bounding-box. For these cases, several relative bigger bounding-boxes are reserved in the step ii, and we exploit those bigger bounding-boxes to further generate a tight bounding-box. Similarly, we choose the biggest bounding-box from step ii, merge ${ }^{1}$ all the boundingboxes whose intersection-over-union (IoU) with the biggest bounding-box are larger than a threshold ( $\left.T_{\text {fusion }}\right) 0.4$, and save the merged bounding-box. And then, we select the biggest one in the rest of bounding-boxes to repeat this procedure, and so on. All the bounding-boxes generated by procedure ii and iii are saved as the pseudo ground truth bounding-boxes.

Since we only use image-level labels to train the WSD, the prediction bounding-boxes are coarse, which leads to the pseudo ground truth bounding-boxes generated by the above three procedures may be inaccurate or contain too much context information. To overcome this issue, we combined the partially labeled bounding-box annotations and the mined bounding-boxes to reinforce the quality of the pseudo ground truth bounding-boxes. Here, we design two different combination methods: (i) hard combination method, and (ii) soft combination method. The ablation study in section V-D will show which method is better for combining the mined boundingboxes and the partially labeled bounding-box annotations.

Hard combination method. One of the simplest methods for combining the mined pseudo ground truth bounding-boxes and the partially labeled bounding-boxes is to stack all the bounding-boxes together directly. The intuition is that, for a labeled instance, there is always an accurate bounding-box to guide the network to generate the detection results.

Soft combination method. The approach of hard combination has one limitation: the inaccurate mined pseudo ground truth bounding-boxes may mislead the network converge to a local optimum, and damage the quality of the mined results. Therefore, we propose a soft combination method. For $k p_{t h}$ pseudo bounding-box $G_{P}$, we first calculate the area of the overlap between $G_{P}$ and all the labeled ground truth bounding-boxes $G$ in $i_{t h}$ training image and then define an interior part score $s_{p}$ :

$$
s_{p}=A\left(G_{P}^{k p} \cap G_{i}^{j l}\right) / A\left(G_{P}^{k p}\right)
$$

where $k p=(1,2, \ldots, K p), K p$ denotes the number of the mined pseudo ground truth bounding-boxes, $j l=$ $(1,2, \ldots, J l), J l$ denotes the number of the labeled ground truth bounding-boxes, and $A$ denotes the area of the boundingboxes. In particular, if $s_{p}$ is larger than a pre-define threshold

\footnotetext{
${ }^{1}$ We choose the minimum top-left coordinate and maximum bottom-right coordinate of the bounding-boxes as the final coordinates.
}

$T_{s_{p}}=0.7$, we think this instance has a labeled ground truth bounding-box, and the mined pseudo ground truth boundingboxes will be discarded. On the contrary, if the $s_{p}$ is smaller than the $T_{s_{p}}$, we think this instance does not have the labeled ground truth bounding-box, and the pseudo mined pseudo ground truth bounding-boxes will be saved.

Once achieving $G^{\prime}$, we train a region proposal network (RPN) to generate the proposals again for further refining the combined pseudo ground truth bounding-boxes. The specific progress is that, for each combined pseudo ground truth bounding-box, we choose all the proposals generated by RPN whose IoU with the combined bounding-box are larger than a threshold $\left(T_{i o u}\right) 0.5$, and then average the pixel coordinates of these selected proposals and save the averaged coordinate as the final pseudo ground truth bounding-boxes, which are used to train an FSD in our pipeline. In this paper, we choose the Fast-RCNN [8] as our FSD.

In Figure 3, we show some results of the pseudo ground truth bounding-boxes mined by our proposed PGTM algorithm, and the corresponding ones generated by the W2F method [1]. As we can see from the comparison, our method can generate higher quality pseudo ground truth boundingboxes than the method in [1].

\section{The Proposed IL Framework}

In the aforementioned WSD, the classification model is used to derive a detector, and the image-level labels are transferred to the instance-level annotations. In general, the classification network usually utilizes the specific pattern of the categories for recognition, and the results are that only a small region of the objects is highlighted instead of the whole body of the objects. In our paper, although partially instancelevel annotations are used to mine the pseudo ground truth bounding-boxes by the PGTM algorithm, we would like to note that the mined pseudo ground truth bounding-boxes are still worse than the bounding-boxes annotated by human. This is because the candidate bounding-box regions in the PGTM procedure are predicted by the WSD. To address this issue, we propose an IL framework which aims at finding the integral object region progressively and producing the tight pseudo ground truth bounding-boxes.

As shown in Figure 4, our proposed IL framework includes three components: WSD, PGTM, and FSD. We first train an WSD by only using the image-level labels, as described in sub-section IV-A. Then, the prediction bounding-boxes from the WSD and the partially annotated bounding-boxes are combined by the proposed PGTM procedure, which can generate the reliable pseudo ground truth bounding-boxes. Finally, the combined bounding-boxes are used to train an FSD. The PGTM procedure and the FSD are iterated until optimal pseudo ground truth bounding-boxes are mined. With the iteration going, the pseudo ground truth bounding-boxes are obtained from coarse to accurate, and the final detection results form the FSD are improved dramatically.

We would like to note that all aforementioned three components and the iterative processes are implemented in the training period. For inference, only the $n_{t h}$ FSD is utilized, 


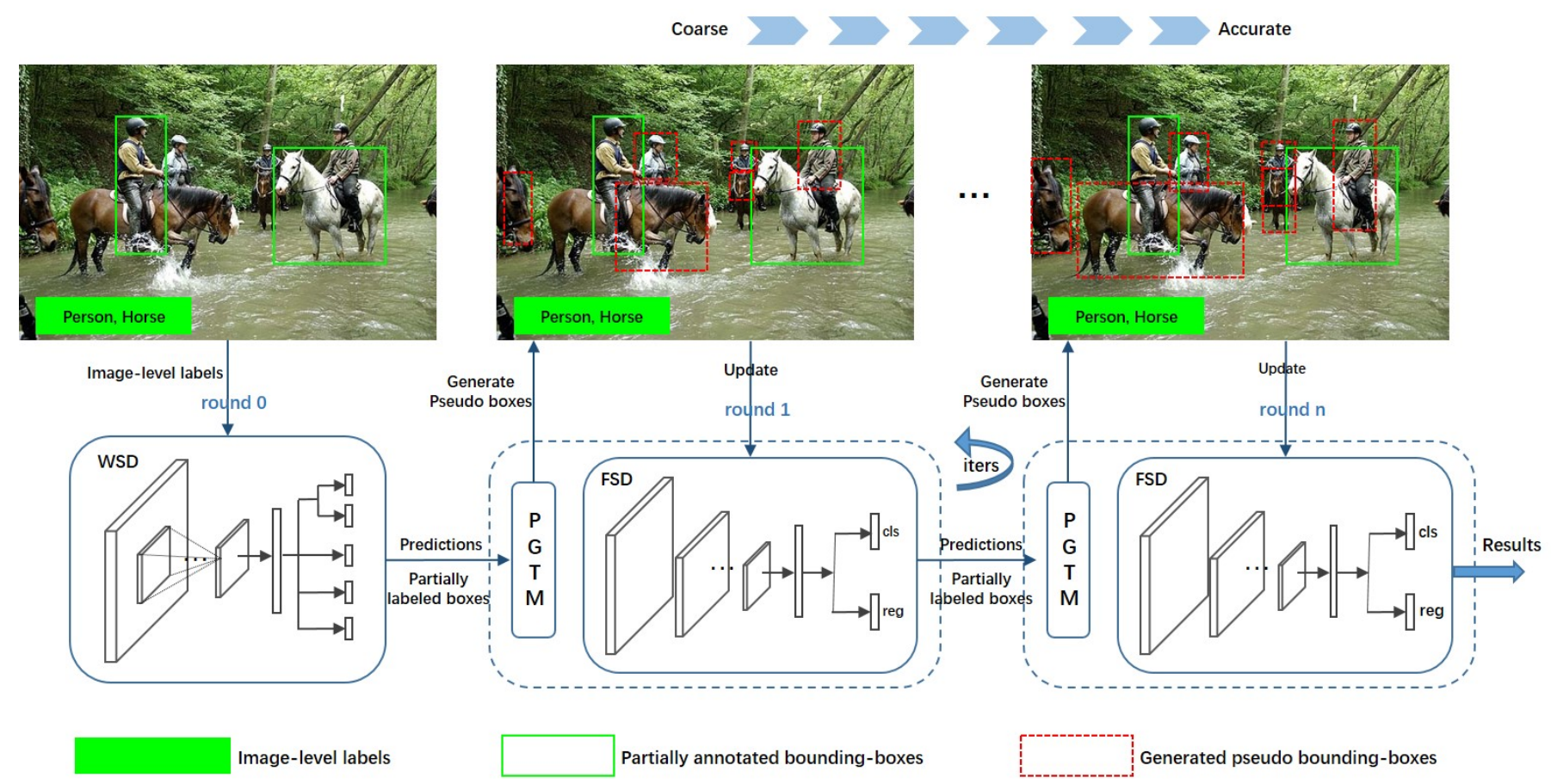

Fig. 4. The pipeline of the proposed IL framework. At first, we train an WSD by only using image-level labels. Then, the prediction bounding-boxes from the WSD and the partially annotated instance-level annotations (i.e. solid green boxes) are inputted to the proposed PGTM procedure, and the outputs are reliable pseudo ground truth bounding-boxes (i.e. dot red boxes). Finally, an FSD is trained under the supervision of the mined pseudo ground truth bounding-boxes. In the following rounds (iterations), the FSD can produce more reliable prediction bounding-boxes, which are combined with the partially annotated instance-level bounding-boxes again to mine the high-quality pseudo ground truth bounding-boxes that further update the FSD. When the PGTM and FSD update iteratively, the pseudo ground truth bounding-boxes are obtained from coarse to accurate, and the performance of the FSD improves steadily. Best seen on the computer, in color and zoomed in.

which is chosen based on the detection performance during training stage, to predict the category and location of the target objects, so the inference process is efficient.

\section{EXPERIMENTS}

\section{A. Experiment Setup}

We evaluate the proposed method on three challenging and widely used benchmarks, i.e. PASCAL VOC 2007, PASCAL VOC 2012, MS COCO. For details about the PASCAL VOC benchmarks and the evaluation metric, please see subsection III-A. In addition, correct location (CorLoc) metric [51] is also employed to evaluate the localization accuracy of the bounding-boxes predicted by our model on the training set. Both of the metrics (i.e. mAP and CorLoc) comply with the PASCAL criterion, where a positive detection result has an IoU $>0.5$ with the ground-truth bounding-boxes. As for $\mathrm{COCO}$ dataset, the standard COCO evaluation metrics are employed to report the detection results.

\section{B. Implementation Details}

When generating the missing bounding-box annotations, the missing rate $m_{r}$ ranges from 0.1 to 0.9 , and the step size is 0.1 . In terms of VOC datasets, the backbone network of all the experiments (e.g. WSD, RPN, FSD) is VGG16 pre-trained on the ImageNet [5]. For data augmentation, we randomly resize the short side of the images to one of five scales $480,576,688$, 864,1200 , and we enforce the long side of the images to be shorter than 2000. Furthermore, we randomly flip the training images in the horizontal direction. In the WSD, we set the total iterations to $70 \mathrm{~K}$. We imperially set the learning rate to 0.001 in the first $40 \mathrm{~K}$ iterations and divided by a factor 10 in the last $30 \mathrm{~K}$ iterations. In addition, we use mini-batch size 2 , and the momentum and weight decay are set to 0.9 and 0.0005 respectively. In the PGTM procedure, we follow the settings in [12] to train the RPN, and for other pre-define parameters please refer to sub-section IV-B. In the IL framework, the settings of the FSD (i.e. Fast-RCNN) are the same as [8], and the iteration times is set to 3 by the ablation studies in subsection V-D.

As for the COCO dataset, the standard training, validation and testing splits are used for training and evaluating the proposed method. Note that we do not train the WSD on COCO dataset and use the model trained on VOC2012 dataset to generate the candidate bounding-box regions.

\section{Comparison of Methods for Generating Missing Bounding- box Annotations}

To validate which one is more effective between the instance-level missing and image-level missing methods for generating the missing bounding-box annotations, we use these two methods to prepare the missing bounding-box annotations respectively, and report the results of Round 1 (i.e. no iteration training) in the IL framework, in which the soft combination method is used in the PGTM procedure. From Figure 5, we can observe that the performance of the image-level missing 
TABLE I

THE PERFORMANCE (AP\%) OF OUR METHODS WITH AND WITHOUT THE PROPOSED COMPONENTS ON THE PASCAL VOC 2007 test SET. WSD+FSD IS OUR METHOD SELECTING THE TOP SCORE PREDICTION FORM THE WSD AS THE PSEUDO GROUND TRUTH BOUNDING-BOXES AS IN [24] TO TRAIN AN FSD. NOTE THAT THE REPORTED RESULTS ARE ACHIEVED AT MISSING RATE $m_{r}=0.9$.

\begin{tabular}{|c|c|c|c|c|c|c|c|c|c|c|c|c|c|c|c|c|c|c|c|c|c|}
\hline ethod & ero & ike & ird & boat & ottle & bus & car & cat & hair & cow & table & dog & horse & $\mathrm{mbi}$ & per & plant & shee & sofa & train & tv & \\
\hline & 4 & 6 & 3 & 87 & 1 & & & & .7 & & 35.0 & & & 4.1 & 12.6 & 23 & 40.0 & & & & 1.6 \\
\hline & & 7 & & & & & & & & & & & & & & 22 & & & & & 5. \\
\hline & & 0.1 & 0.5 & 31.9 & 4 & 0 & & 7 & 3 & & 49.4 & 65.9 & 57.2 & 2 & & 23.8 & .8 & 7 & 0 & 3 & 2 \\
\hline & 4.1 & 66.2 & 50.3 & .2 & 16.5 & 9 & & & 21.4 & & 53.4 & 70.6 & .6 & & & 22.4 & 0.5 & 0 & & .3 & \\
\hline WSD+PG & 64.2 & 69.6 & 50.4 & 32.3 & 17.0 & 73.4 & 71.5 & 73.8 & 22.5 & 58.4 & 55.5 & 72.0 & 60.9 & 67.6 & 26.3 & 24.4 & 50.1 & 63.1 & 64.5 & 57.7 & \\
\hline WSD+PGTM(soft)+FSD+IL & 64.4 & 72.2 & 54.5 & 37.7 & 17.3 & 75.6 & 73.5 & 71.8 & 27.7 & 64.8 & 55.2 & 65.4 & 64.1 & 66.5 & 37.6 & 24.2 & 56.3 & 56.7 & 65.6 & 60.5 & \\
\hline
\end{tabular}

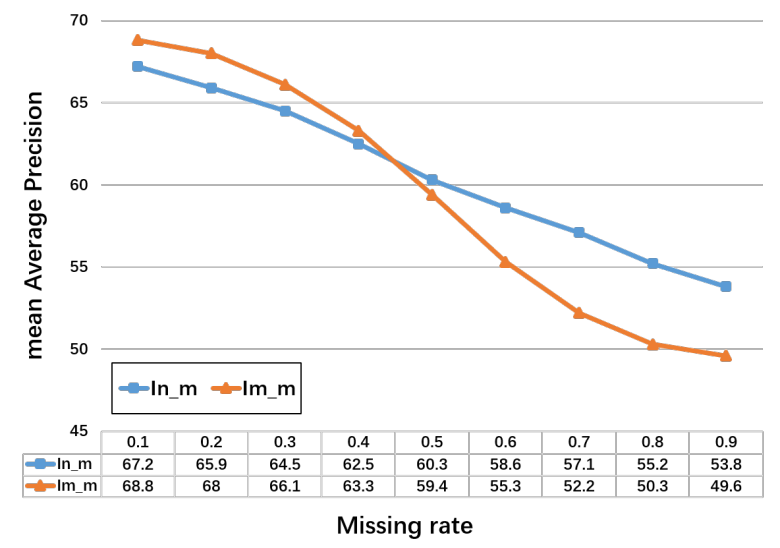

Fig. 5. The performance (mAP) of the different methods (i.e. instancelevel missing method and image-level missing method) for generating the missing bounding annotations on PASCAL VOC 2007 dataset, where In_m denotes the instance-level missing method and Im_m denotes the image-level missing method. Note that the presented results come from Round 1 in the IL framework, and we use the soft combination method in the PGTM procedure.

method is better than the instance-level missing method at low missing rates (i.e. from 0.1 to 0.4 ). On the contrary, the performance of the instance-level missing method surpasses the image-level missing method as the missing rate increases. For instance, mAP of the instance-level missing method is $53.8 \%$ compared to $49.6 \%$ in the image-level missing method when the missing rate equals 0.9 . From subsection III-B, we know that the instance-level missing method corresponds to the setting of the partially labeled bounding-box annotations in Figure 1 (a) (i.e. each image in the training data has instance-level annotations, but only the simple instances are labeled), and the image-level missing method corresponds to the setting of the partially labeled bounding-box annotations in Figure 1 (b) (i.e. only part of images in the training data have instance-level annotations where all instances in these images are annotated, and other images have no instancelevel annotations). The above experiments and analyses make clear which one is the most effective way to label the massive images with limited labors and funds, and the answer is to annotate the obvious (or simple) instances for each training images instead of annotating all of the instances of only part of the training images. In our paper, we focus on object detection under the few partially labeled bounding-box annotations, so we use the instance-level missing method to generate the missing bounding-box annotations in the following experiments.
TABLE II

THE MAP PERFORMANCE OF THE PROPOSED IL FRAMEWORK WITH DIFFERENT ITERATION TIMES ON THE PASCAL VOC 2007 test SET, WHERE N DENOTES THE NUMBER OF ITERATIONS, AND THE MISSING RATE EQUALS 0.9 .

\begin{tabular}{l|cccc}
\hline Iterations(n) & 1 & 2 & 3 & 4 \\
\hline mAP\% & 53.8 & 55.2 & 55.6 & 55.5 \\
\hline
\end{tabular}

\section{Ablation Studies}

Effectiveness of the PGTM procedure. We validate the effectiveness of our proposed PGTM procedure by conducting the ablation experiments between WSD+FSD and WSD+PGTM+FSD at a missing rate $m_{r}=0.9$. Table I (the 2nd and 4th/5th rows) indicates that our proposed PGTM with hard bounding-box combination and soft combination methods improve the mAP by $5.9 \%$ and $7.0 \%$, respectively. We thank the high-quality pseudo ground truth boundingboxes mined by the proposed PGTM procedure for this huge improvement, which confirms the positive influence of the PGTM procedure on the pipeline of the missing boundingboxes object detection. From Table I (the 4th and 5th rows), we can see that the performance of the soft combination method is superior to the hard combination method (52.7\% vs. 53.8\%) in the PGTM procedure. So we use the soft combination method to merge the mined pseudo ground truth boundingboxes and the partially labeled bounding-boxes in all the following experiments unless otherwise stated.

Effectiveness of the IL framework. Table II shows the comparison of the mAP with different iteration times $n=$ $(1,2,3,4)$ in the IL framework on the PASCAL VOC 2007 test set. From Table II, we can find that the performance with no iteration operation (i.e. $n=1$ ) is $53.8 \%$. By performing the iterative training, the performance increases dramatically (from $53.8 \%$ to $55.2 \%$ ), which demonstrates that the IL framework improves the quality of the mined pseudo ground truth bounding-boxes in a positive trend. As the iterative training goes on, the detection network converges to one point (i.e. the performance is about 55.6\%). However, when the number of iterations reaches 4, the performance does not change any more. So we set iteration times of the IL framework to 3 (i.e. $n=3$ ) for all the experiments in our paper. In addition, to validate the contribution of the IL framework, we conduct an experiment by adding the IL operation to one of our experiment settings (i.e. WSD+PGTM(soft)+FSD). From Table I (the 5th and 6th rows), we can observe that the IL framework improves the mAP from $53.8 \%$ to $55.6 \%$, which further proves the effectiveness of the IL framework. 
TABLE III

THE PERFORMANCE (AP\%) OF OUR PROPOSED METHOD AND OTHER STATE-OF-THE-ART METHODS ON THE PASCAL VOC 2007 test SET. THE UPPER PART IS SOME FULLY-SUPERVISED DETECTORS AND THE BOTTOM PART IS SOME WEAKLY-SUPERVISED DETECTORS.

\begin{tabular}{|c|c|c|c|c|c|c|c|c|c|c|c|c|c|c|c|c|c|c|c|c|c|}
\hline Method & ero & bike & bird & boat & bottle & bus & car & cat & chair & cow & table & $\operatorname{dog}$ & horse & mbike & person & plant & sheep & sofa & train & tv & $\overline{\mathrm{mAP}}$ \\
\hline YOLO [17] & 3.4 & 77.6 & 65.2 & 55.0 & 42.4 & 76.9 & 77.3 & 80.5 & 45.4 & 69.4 & 72.6 & 76.5 & 80.1 & 77.0 & 72.3 & 42.9 & 63.3 & 64.8 & 78.7 & 66.6 & 67.9 \\
\hline SSD [14] & 3.4 & 77.5 & 64.1 & 59.0 & 3.9 & 75.2 & 80.8 & 78.5 & 46.0 & 67.8 & 69.2 & 76.6 & 82.1 & 77.0 & 72.5 & 41.2 & 64.2 & 69.1 & 78.0 & 8.5 & 68.0 \\
\hline CNN [8] & 6 & 79.0 & 68.6 & 57.0 & & 79.5 & 78.6 & 1.9 & 48.0 & 74.0 & 67.4 & 80.5 & & 74.1 & 69.6 & 31.8 & & 68.4 & 15.3 & & 58.1 \\
\hline Faster-RCNN [12] & 0.0 & 80.6 & 70.1 & 57.3 & 49.9 & 78.2 & 80.4 & 2.0 & 52.2 & 75.3 & 67.2 & 80.3 & 79.8 & 75.0 & 76.3 & 39.1 & 68.3 & 67.3 & 1.1 & 67.6 & 69.9 \\
\hline Ours_ $m_{r}=0.1$ & 2 & 78.5 & 66.7 & 5.7 & 7.7 & 78.8 & 79.9 & 1.1 & 41.1 & 76.2 & 66.3 & 82.7 & 78.2 & 72.2 & 6.1 & 30.7 & 6.7 & 3 & 6.7 & 4.7 & 7.1 \\
\hline Ours_ $m_{r}=0$ & .0 & 77.2 & 65.3 & 6.0 & 6 & 77.0 & 8.0 & 6 & 0.4 & 5 & 8 & 81.1 & 76.6 & 2.4 & 3 & 31.0 & 0 & 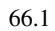 & .2 & 4.0 & 6.0 \\
\hline Ours_ $m_{r}=0.3$ & 2.5 & 76.6 & 64.2 & 55.3 & .8 & 77.7 & 78.2 & 3 & 37.9 & 4.4 & 65.1 & 80.0 & 5.6 & 2.1 & 62.9 & 30.1 & 4.1 & 3 & 4.6 & 3.2 & 5.3 \\
\hline Ours_ $m_{r}=0.4$ & 1.9 & 76.0 & 61.9 & 44.4 & 3.6 & 76.9 & 77.6 & 8.9 & 30.2 & 72.5 & 64.5 & 78.2 & 74.5 & 0.1 & 58.7 & 28.8 & 3.4 & 8.0 & 72.5 & 60.6 & 3.2 \\
\hline Ours_ $m_{r}=0.5$ & 2.9 & 75.9 & 60.7 & 44.9 & 31.4 & 78.2 & 77.5 & 80.0 & 26.9 & 71.8 & 64.0 & 76.8 & 72.7 & 69.7 & 53.6 & 27.4 & 62.6 & 69.2 & 71.3 & 59.2 & 62.3 \\
\hline$n_{r}=0.6$ & 1.4 & 70.9 & 58.7 & 43.6 & 0.8 & 76.6 & 76.4 & .3 & 27.1 & 66.9 & 63.1 & 75.4 & 72.0 & 69.9 & 45.8 & 26.1 & 59.3 & 67.4 & 68.5 & 59.0 & 50.4 \\
\hline Ours_ $m_{r}=0.7$ & 4 & 71.6 & 54.0 & 42.8 & 26.6 & 74.2 & 75.9 & 77.7 & 22.2 & 66.1 & 58.4 & 74.5 & 70.0 & 69.6 & 36.6 & 24.3 & 58.6 & 8 & 56.3 & 58.3 & 58.1 \\
\hline Ours_ $m_{r}=0.8$ & 6.6 & 71.2 & 53.9 & 40.1 & 5.4 & 73.9 & 74.0 & 75.5 & 23.4 & 65.2 & 57.1 & 72.3 & 68.5 & 68.2 & 36.5 & 23.9 & 57.5 & 63.2 & 8 & 57.9 & 57.0 \\
\hline Ours_ $m_{r}=0.9$ & 4.4 & 72.2 & 54.5 & 37.7 & 17.3 & 75.6 & 73.5 & 71.8 & 27.7 & 64.8 & 55.2 & 65.4 & 64.1 & 66.5 & 37.6 & 24.2 & 56.3 & 56.7 & 65.6 & 60.5 & 55.6 \\
\hline OICR [24] & 58.5 & 63.0 & 35.1 & 16.9 & 17.4 & 63.2 & 60.8 & 34.4 & 8.2 & 49.7 & 41.0 & 31.3 & 51.9 & 64.8 & 13.6 & 23.1 & 41.6 & 48.4 & 58.9 & 58.7 & 42. \\
\hline M [27] & & 66.9 & 34.2 & 29.1 & . & 68.8 & 68.1 & 0 & 25.0 & 65.6 & 45.3 & 53.2 & 49 & 68.6 & 2. & 25.4 & 52.5 & 56.8 & 62.1 & 57.1 & 47. \\
\hline & & & 50.1 & 8 & & & & & & & 4 & 4 & & & & 27 & 53 & 56.1 & 5 & 58.2 & 7. \\
\hline & & & & & & & & & & & & & & & & 22 & & & & 55.1 & 1. \\
\hline & & & & & & & & & & & & & & & & 23.6 & & & & 56.6 & 44. \\
\hline ang et. al [55] & 0.3 & 66.2 & 5.0 & & 6.6 & 68.1 & & & & 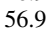 & & 33 & 62 & 2 & 20.6 & 29.0 & 49.0 & & & 58.4 & 47. \\
\hline & & 70.1 & 50.5 & 31.9 & & 72.0 & 67.8 & & 23.3 & & & 65.9 & 57 & 67 & 27.6 & 23.8 & 51.8 & & & 62.3 & 52. \\
\hline Dual-Network [44] & 62.5 & 54.6 & 44.3 & 12.9 & 12.7 & 63.8 & 60.6 & 25.0 & 5.4 & 48.0 & 49.3 & 58.7 & 66.6 & 63.5 & 8.5 & 17.3 & 40.7 & 59.4 & 53.9 & 51.4 & 43. \\
\hline
\end{tabular}

TABLE IV

THE PERFORMANCE (MAP\%) OF OUR PROPOSED METHOD AND OTHER STATE-OF-THE-ART METHODS ON THE PASCAL VOC 2012 test SET. SIMILARLY, THE UPPER PART IS SOME FULLY-SUPERVISED DETECTORS AND THE BOTTOM PART IS SOME WEAKLY-SUPERVISED DETECTORS. THE PER-CLASS RESULTS CAN BE FOUND IN SUPPLEMENTARY MATERIALS.

\begin{tabular}{|c|c|c|c|}
\hline Method & $\mathrm{mAP}$ & Method & mAP \\
\hline YOLO [17] & 73.4 & SSD [14] & 72.4 \\
\hline Fast-RCNN [8] & 68.4 & Faster-RCNN [12] & 70.4 \\
\hline Ours $\_m_{r}=0.1$ & 67.8 & Ours $\_m_{r}=0.6$ & 61.5 \\
\hline Ours_ $m_{r}=0.2$ & 66.9 & Ours_ $m_{r}=0.7$ & 59.6 \\
\hline Ours_ $m_{r}=0.3$ & 65.8 & Ours_ $m_{r}=0.8$ & 58.8 \\
\hline Ours_ $m_{r}=0.4$ & 64.3 & Ours_ $m_{r}=0.9$ & 56.3 \\
\hline Ours_ $m_{r}=0.5$ & 63.1 & & \\
\hline OICR [24] & 37.9 & MELM [27] & 42.4 \\
\hline Zhang et. al [52] & 42.9 & GALfWSD [53] & 43.1 \\
\hline TS2C [54] & 40.0 & Tang et. al [55] & 43.4 \\
\hline $\mathrm{W} 2 \mathrm{~F}[1]$ & 47.8 & Dual-Network [44] & 36.6 \\
\hline
\end{tabular}

Improvement over W2F. Our proposed method (i.e. PGTM) is modified from our previous work $\mathrm{W} 2 \mathrm{~F}$, and please refer to subsection IV-B for the details of the modification. Compared to W2F, as shown in Table I (the 3 rd and 6th rows), the proposed method improves the mAP by $3.2 \%$ absolutely (i.e. from $52.4 \%$ to $55.6 \%$ ) when the missing rate equals 0.9 . There are two reasons for this huge improvement, and the first one is that some bounding-boxes annotated by human are combined to mine the pseudo ground truth bounding-boxes and further used to train the FSD. The second one is that the quality of the pseudo ground truth bounding-boxes mined by our proposed PGTM is better than the ones found by W2F, which is the main reason for the improvement in our opinion.

\section{E. Comparisons with State-of-the-art Methods}

Results on VOC2007. Table III shows the AP performance of our method and other state-of-the-art methods on the VOC 2007 test set. Form Table III, we can see that we achieve $55.6 \% \mathrm{mAP}$ at a high missing rate (missing rate $m_{r}=$ 0.9 ), outperforming the highest performance of the weaklysupervised methods (i.e. the bottom part of Table) by $3.2 \%$
TABLE V

DETECTION AND LOCALIZATION COMPARISONS TO THE STATE-OF-THE-ART METHODS ON PASCAL VOC 2007 AND VOC 2012 trainval SET IN TERMS OF CORLOC (\%) METRICS, RESPECTIVELY. THE PER-CLASS RESULTS CAN BE FOUND IN SUPPLEMENTARY MATERIALS.

\begin{tabular}{l|cc||l|cc}
\hline Method & 07 & 12 & Method & 07 & 12 \\
\hline Ours_m $m_{r}=0.1$ & 79.2 & 82.6 & Ours_ $m_{r}=0.6$ & 75.6 & 77.1 \\
Ours_ $m_{r}=0.2$ & 78.1 & 81.2 & Ours_ $m_{r}=0.7$ & 74.7 & 76.1 \\
Ours_m $m_{r}=0.3$ & 77.8 & 79.6 & Ours_ $m_{r}=0.8$ & 74.0 & 75.0 \\
Ours_ $m_{r}=0.4$ & 76.9 & 78.8 & Ours_ $m_{r}=0.9$ & 72.8 & 73.0 \\
Ours_ $m_{r}=0.5$ & 76.4 & 77.9 & & & \\
\hline OICR [24] & 60.6 & 62.1 & MELM [27] & 61.4 & - \\
Zhang et. al [52] & 61.2 & 61.5 & GALfWSD [53] & 68.1 & 67.2 \\
TS2C [54] & 61.0 & 64.4 & Tang et. al [55] & 66.9 & 67.2 \\
W2F [1] & 70.3 & 69.4 & Dual-Network [44] & 69.0 & 61.4 \\
\hline
\end{tabular}

mAP absolutely. When comparing with the fully-supervised methods (i.e. the upper part of the Table), our method can get comparable performance (e.g. $62.3 \%$ at $m_{r}=0.5$ vs. $68.1 \%$ in [8], and $65.3 \%$ at $m_{r}=0.3 v s .68 .1 \%$ in [8]). In addition, Table $\mathrm{V}$ show the detection and localization comparisons to state-of-the-art methods on PASCAL VOC 2007 trainval set in term of CorLoc metrics. From Table V, we can observe that, our CorLoc performance surpasses the second highest method by $2.5 \%$ absolutely when the missing rate equals 0.9 . Based on the comparison results, we can confirm that our proposed method can exactly narrow the gap between the fully-supervised and weakly-supervised methods for object detection. Moreover, our method can solve the missing boundingbox object detection problem successfully.

Results on VOC20012. Table IV and Table V show the mAP and CorLoc performance on VOC 2012 test and trainval sets, respectively. Similarly, our proposed method at a high missing rate (i.e. missing rate $m_{r}=0.9$ ) surpasses the best weakly-supervised methods by $8.5 \%$ in terms of mAP, and achieves the comparable performance when comparing with the fully-supervised methods. Meanwhile, the CorLoc of our method outperforms other methods by a large margin. More importantly, the performance of our method exceeds the results largely achieved by directly using the missing boundingbox annotations to train FSDs, which further confirms the 
TABLE VI

THE PERFORMANCE (MAP) OF THE PROPOSED METHOD AND OTHER STATE-OF-THE-ART METHODS ON COCO test - dev SUBSET.

\begin{tabular}{l|l|l}
\hline Methods & Backbone & mAP \\
\hline Ours_ $m_{r}=0.9$ & ResNet-50-C4 & 15.6 \\
Ours_ $m_{r}=0.7$ & ResNet-50-C4 & 19.4 \\
Ours_ $m_{r}=0.5$ & ResNet-50-C4 & 23.3 \\
Ours_ $m_{r}=0.3$ & ResNet-50-C4 & 26.6 \\
Ours_ $m_{r}=0.1$ & ResNet-50-C4 & 28.3 \\
\hline SSD512 [14] & VGG16 & 26.8 \\
YOLO9000 [17] & Darknet-19 & 21.6 \\
Faster RCNN [12] & ResNet-101-C4 & 34.9 \\
FPN [41] & ResNet-101-FPN & 36.2 \\
Mask RCNN [13] & ResNeXt-101-FPN & 39.8 \\
\hline
\end{tabular}

\section{effectiveness of our proposed method.}

Results on COCO dataset. To further validate the effectiveness of the proposed method, we conduct the comparison experiments on COCO dataset. Since we do not find any WSD uses the standard $\mathrm{COCO}$ evaluation metrics to evaluate their method, we just compare the achieved results with some fullysupervised state-of-the-art methods [12]-[14], [17], [41]. As shown in Table VI, our proposed method achieves competitive performance on the large-scale detection dataset.

\section{CONClusion AND Future Work}

In this paper, we first formulate object detection task under supervision of partially annotated bounding-boxes as a missing bounding-boxes object detection problem. Different from previous semi-supervised methods, our proposed method successfully combines the advantages of weakly-supervised and fully-supervised methods. To address the missing boundingboxes problem, we develop a pseudo ground truth mining (PGTM) procedure to automatically find the pseudo ground truth bounding-boxes for those unlabeled instances in the training data, and then combine the partially labeled annotations and the mined pseudo ground truth bounding-boxes to train an object detector. Furthermore, an incremental learning (IL) framework is proposed to gradually incorporate the results of the trained detector to improve the performance of the missing bounding-boxes object detection. As a by-product, we reveal how the missing bounding-boxes will impact on an FSD, and find an effective way to label the massive images with limited labors and funds. Under few labeled boundingbox annotations (i.e. $m_{r}=0.9$ ), the proposed method can achieve 55.6\% and 56.3\% mAP on PASCAL VOC 2007 and 2012, respectively, which outperforms the previous state-ofthe-art weakly-supervised methods by a large margin, and gets comparable performance to some fully-supervised methods.

In the future, we will extend our method to detect the objects with fewer labeled bounding-box annotations (i.e. only several bounding-boxes per category).

\section{REFERENCES}

[1] Y. Zhang, Y. Bai, M. Ding, Y. Li, and B. Ghanem, "W2f: A weaklysupervised to fully-supervised framework for object detection," in IEEE CVPR, 2018, pp. 928-936.

[2] — "Weakly-supervised object detection via mining pseudo ground truth bounding-boxes," Pattern Recognition, vol. 84, pp. 68-81, 2018.

[3] Y. Bai, Y. Zhang, M. Ding, Y. Li, and B. Ghanem, "Sod-mtgan: Small object detection via multi-task generative adversarial network," in $E C C V$, 2018.
[4] K. He, X. Zhang, S. Ren, and J. Sun, "Deep residual learning for image recognition," in IEEE CVPR, 2016, pp. 770-778.

[5] A. Krizhevsky, I. Sutskever, and G. E. Hinton, "Imagenet classification with deep convolutional neural networks," in NIPs, 2012, pp. 10971105.

[6] C. Szegedy, W. Liu, Y. Jia, P. Sermanet, S. Reed, D. Anguelov, D. Erhan, V. Vanhoucke, and A. Rabinovich, "Going deeper with convolutions," in IEEE CVPR, 2015, pp. 1-9.

[7] P. Sermanet, D. Eigen, X. Zhang, M. Mathieu, R. Fergus, and Y. LeCun, "Overfeat: Integrated recognition, localization and detection using convolutional networks," arXiv preprint arXiv:1312.6229, 2013.

[8] R. Girshick, "Fast r-cnn," in IEEE ICCV, 2015, pp. 1440-1448.

[9] R. Girshick, J. Donahue, T. Darrell, and J. Malik, "Rich feature hierarchies for accurate object detection and semantic segmentation," in IEEE CVPR, 2014, pp. 580-587.

[10] J. Dai, Y. Li, K. He, and J. Sun, "R-fcn: Object detection via regionbased fully convolutional networks," in NIPs, 2016, pp. 379-387.

[11] T. Kong, F. Sun, A. Yao, H. Liu, M. Lu, and Y. Chen, "Ron: Reverse connection with objectness prior networks for object detection," in IEEE CVPR, vol. 1, 2017, p. 2.

[12] S. Ren, K. He, R. Girshick, and J. Sun, "Faster r-cnn: towards real-time object detection with region proposal networks," IEEE TPAMI, no. 6, pp. 1137-1149, 2017.

[13] K. He, G. Gkioxari, P. Dollár, and R. Girshick, "Mask r-cnn," in IEEE ICCV. IEEE, 2017, pp. 2980-2988.

[14] W. Liu, D. Anguelov, D. Erhan, C. Szegedy, S. Reed, C.-Y. Fu, and A. C. Berg, "Ssd: Single shot multibox detector," in ECCV. Springer, 2016, pp. 21-37.

[15] C.-Y. Fu, W. Liu, A. Ranga, A. Tyagi, and A. C. Berg, "Dssd: Deconvolutional single shot detector," preprint arXiv:1701.06659, 2017.

[16] J. Redmon, S. Divvala, R. Girshick, and A. Farhadi, "You only look once: Unified, real-time object detection," in IEEE CVPR, 2016, pp 779-788.

[17] J. Redmon and A. Farhadi, "Yolo9000: better, faster, stronger," arXiv preprint, 2017.

[18] Y. LeCun, L. Bottou, Y. Bengio, and P. Haffner, "Gradient-based learning applied to document recognition," Proceedings of the IEEE, vol. 86, no. 11, pp. 2278-2324, 1998.

[19] M. Everingham, L. Van Gool, C. K. Williams, J. Winn, and A. Zisserman, "The pascal visual object classes (voc) challenge," International journal of computer vision, vol. 88, no. 2, pp. 303-338, 2010.

[20] T.-Y. Lin, M. Maire, S. Belongie, J. Hays, P. Perona, D. Ramanan, P. Dollár, and C. L. Zitnick, "Microsoft coco: Common objects in context," in ECCV. Springer, 2014, pp. 740-755.

[21] R. G. Cinbis, J. Verbeek, and C. Schmid, "Weakly supervised object localization with multi-fold multiple instance learning," IEEE TPAMI, vol. 39, no. 1, pp. 189-203, 2017.

[22] V. Kantorov, M. Oquab, M. Cho, and I. Laptev, "Contextlocnet: Contextaware deep network models for weakly supervised localization," in ECCV. Springer, 2016, pp. 350-365.

[23] H. Bilen and A. Vedaldi, "Weakly supervised deep detection networks," in IEEE CVPR, 2016, pp. 2846-2854.

[24] P. Tang, X. Wang, X. Bai, and W. Liu, "Multiple instance detection network with online instance classifier refinement," in CVPR, 2017.

[25] K. Wang, X. Yan, D. Zhang, L. Zhang, and L. Lin, "Towards humanmachine cooperation: Self-supervised sample mining for object detection," in IEEE CVPR, June 2018.

[26] X. Zhang, Y. Wei, J. Feng, Y. Yang, and T. Huang, "Adversarial complementary learning for weakly supervised object localization," in IEEE CVPR, 2018

[27] F. Wan, P. Wei, J. Jiao, Z. Han, and Q. Ye, "Min-entropy latent model for weakly supervised object detection," in IEEE CVPR, 2018, pp. 12971306.

[28] Z. Yan, J. Liang, W. Pan, J. Li, and C. Zhang, "Weakly-and semisupervised object detection with expectation-maximization algorithm," preprint arXiv:1702.08740, 2017

[29] Y. Tang, J. Wang, X. Wang, B. Gao, E. Dellandréa, R. Gaizauskas, and L. Chen, "Visual and semantic knowledge transfer for large scale semi-supervised object detection," IEEE TPAMI, 2017.

[30] P. K. Rhee, E. Erdenee, S. D. Kyun, M. U. Ahmed, and S. Jin, "Active and semi-supervised learning for object detection with imperfect data," Cognitive Systems Research, vol. 45, pp. 109-123, 2017.

[31] C. Rosenberg, M. Hebert, and H. Schneiderman, "Semi-supervised selftraining of object detection models." in WACV/MOTION, 2005, pp. 2936. 
[32] I. Misra, A. Shrivastava, and M. Hebert, "Watch and learn: Semisupervised learning for object detectors from video," in IEEE CVPR, 2015, pp. 3593-3602.

[33] Y. Yang, G. Shu, and M. Shah, "Semi-supervised learning of feature hierarchies for object detection in a video," in IEEE CVPR, 2013, pp. $1650-1657$.

[34] P. Carbonetto, G. Dorkó, C. Schmid, H. Kück, and N. De Freitas, "A semi-supervised learning approach to object recognition with spatial integration of local features and segmentation cues," in Toward CategoryLevel Object Recognition. Springer, 2006, pp. 277-300.

[35] J. Redmon and A. Farhadi, "Yolov3: An incremental improvement," preprint arXiv:1804.02767, 2018

[36] Z. Li and F. Zhou, "Fssd: Feature fusion single shot multibox detector," preprint arXiv:1712.00960, 2017

[37] L. Zheng, C. Fu, and Y. Zhao, "Extend the shallow part of single shot multibox detector via convolutional neural network," preprint arXiv:1801.05918, 2018.

[38] J. R. Uijlings, K. E. Van De Sande, T. Gevers, and A. W. Smeulders, "Selective search for object recognition," International journal of computer vision, vol. 104, no. 2, pp. 154-171, 2013.

[39] K. He, X. Zhang, S. Ren, and J. Sun, "Spatial pyramid pooling in deep convolutional networks for visual recognition," in ECCV. Springer, 2014, pp. 346-361.

[40] B. Singh, H. Li, A. Sharma, and L. S. Davis, "R-fen-3000 at 30fps: Decoupling detection and classification," preprint arXiv:1712.01802, 2017.

[41] T.-Y. Lin, P. Dollár, R. B. Girshick, K. He, B. Hariharan, and S. J. Belongie, "Feature pyramid networks for object detection." in CVPR, vol. 1 , no. 2, 2017, p. 3.

[42] B. Zhou, A. Khosla, A. Lapedriza, A. Oliva, and A. Torralba, "Learning deep features for discriminative localization," in IEEE CVPR, 2016, pp. 2921-2929.

[43] Y. Wei, J. Feng, X. Liang, M.-M. Cheng, Y. Zhao, and S. Yan, "Object region mining with adversarial erasing: A simple classification to semantic segmentation approach," in IEEE CVPR, vol. 1, no. 2, 2017, p. 3.

[44] X. Dong, D. Meng, F. Ma, and Y. Yang, "A dual-network progressive approach to weakly supervised object detection," in Proceedings of the 2017 ACM on Multimedia Conference, 2017, pp. 279-287.

[45] J. Hoffman, S. Guadarrama, E. S. Tzeng, R. Hu, J. Donahue, R. Girshick, T. Darrell, and K. Saenko, "Lsda: Large scale detection through adaptation," in NIPs, 2014, pp. 3536-3544.

[46] J. Hoffman, D. Pathak, T. Darrell, and K. Saenko, "Detector discovery in the wild: Joint multiple instance and representation learning," in IEEE CVPR, 2015, pp. 2883-2891.

[47] Y. Tang, J. Wang, B. Gao, E. Dellandréa, R. Gaizauskas, and L. Chen, "Large scale semi-supervised object detection using visual and semantic knowledge transfer," in IEEE CVPR, 2016, pp. 2119-2128.

[48] L. Shao, F. Zhu, and X. Li, "Transfer learning for visual categorization: A survey," IEEE transactions on neural networks and learning systems, vol. 26, no. 5, pp. 1019-1034, 2015.

[49] M. Rochan and Y. Wang, "Weakly supervised localization of novel objects using appearance transfer," in IEEE CVPR, 2015, pp. 43154324.

[50] K. Kumar Singh, F. Xiao, and Y. Jae Lee, "Track and transfer: Watching videos to simulate strong human supervision for weakly-supervised object detection," in IEEE CVPR, 2016, pp. 3548-3556.

[51] T. Deselaers, B. Alexe, and V. Ferrari, "Weakly supervised localization and learning with generic knowledge," International journal of computer vision, vol. 100, no. 3, pp. 275-293, 2012.

[52] X. Zhang, J. Feng, H. Xiong, and Q. Tian, "Zigzag learning for weakly supervised object detection," in IEEE CVPR, 2018.

[53] Y. Shen, R. Ji, S. Zhang, W. Zuo, and Y. Wang, "Generative adversarial learning towards fast weakly supervised detection," in IEEE CVPR, 2018, pp. 5764-5773.

[54] Y. Wei, Z. Shen, B. Cheng, H. Shi, J. Xiong, J. Feng, and T. Huang, "Ts2c: Tight box mining with surrounding segmentation context for weakly supervised object detection," preprint arXiv:1807.04897, 2018.

[55] P. Tang, X. Wang, A. Wang, Y. Yan, W. Liu, J. Huang, and A. Yuille, "Weakly supervised region proposal network and object detection," in ECCV, 2018, pp. 352-368.

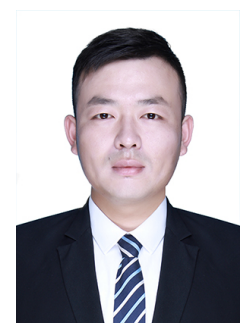

Yongqiang Zhang received the MS degree in instrument science and technology from Harbin Institute of Technology (HIT), Harbin, China, in 2013. He is currently a $\mathrm{PhD}$ student from the Harbin Institute of Technology (HIT), and he worked at King Abdullah University of Science and Technology (KAUST) as a visiting student from 2017 to 2018 . His research areas are computer vision, pattern recognition, machine learning, and deep learning. His research interests mainly include face detection, weakly/fullysupervised object detection, activity detection, image and video understanding in the real-world.

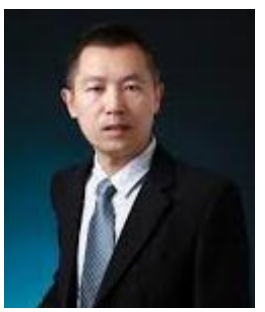

Mingli Ding received the $\mathrm{BS}$, MS and $\mathrm{PhD}$ degrees in instrument science and technology from Harbin Institute of Technology (HIT), Harbin, China, in 1996, 1997 and 2001, respectively. He worked as a visiting scholar in France from 2009 to 2010. Currently, he is a professor in the School of Electrical Engineering and Automation at Harbin Institute of Technology. Prof. Dings research interests are intelligence tests and information processing, automation test technology, computer vision, and machine learning. He has published over 40 papers in peerreviewed journals and conferences.

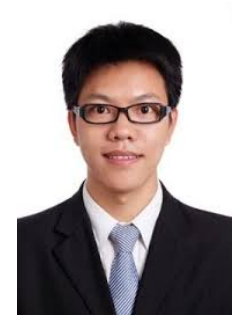

Yancheng Bai is an associate professor in Pattern Recognition and Intelligent System at Institute of Software, Chinese Academy of Sciences, Beijing, China. He received the MS degree from Sichuan university, Sichuan, China, in 2009. He received the $\mathrm{PhD}$ degree from Institute of Automation, Chinese Academy of Sciences, Beijing, China, in 2014. He worked as a post doctoral at King Abdullah University of Science and Technology (KAUST) from 2016 to 2018. His research interests include computer vision, pattern recognition, artificial intelligence, machine learning, and deep learning.

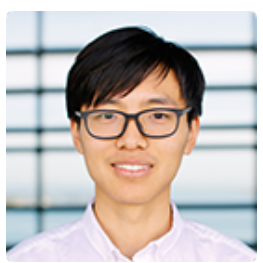

Mengmeng $\mathrm{Xu}$ is an MS student in the CEMSE division and a member of the Visual Computing Center at King Abdullah University of Science and Technology (KAUST), Thuwal, Saudi Arabia. He received the BS degree from Zhejiang university, Zhejiang, China, in 2017. He focus on interesting research problems that arise in computer vision, including activity recognition/detection, robust representations of objects for tracking and recognition, scene understanding from 3D data, image annotation, etc. Since his goal is to make sense of images and videos especially at large-scales, he also often end up developing new machine learning and optimization methods to help him achieve this goal.

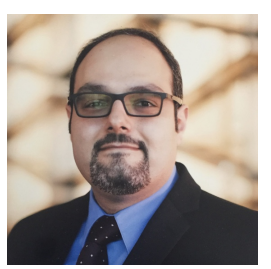

Bernard Ghanem is currently an associate professor in the CEMSE division and a member of the Visual Computing Center at King Abdullah University of Science and Technology (KAUST), Thuwal, Saudi Arabia. Before that, he was a senior research scientist at the University of Illinois Urbana-Champaign (UIUC) in Singapore, where he still holds an adjunct position. He heads projects that develop algorithms in computer vision, machine learning, and optimization geared towards real-world applications, including semantic video analysis in sports and automated surveillance, content-based image retrieval, large-scale activity recognition, and 2D/3D scene understanding. He received his Bachelors degree in Computer and Communications Engineering from the American University of Beirut (AUB) in 2005 and his $\mathrm{MS} / \mathrm{PhD}$ in Electrical and Computer Engineering from UIUC in 2010. His work has received several awards and honors, including the Henderson Graduate Award from UIUC, two consecutive CSE fellowship awards from UIUC, a Best Paper Award (CVPRW 2013), a two-year KAUST Seed Fund, and a Google Faculty Research Award in 2015. He became a area chair of IEEE Conference on Computer Vision and Pattern Recognition (CVPR) in 2017. He has co-authored more than 70 peer reviewed conference and journal papers in his field, as well as, 4 patents. $\mathrm{He}$ is also a co-founder of AutoScount Inc. that provides automated solutions for sports video analytics. 